Bull. Mater. Sci., Vol. 22, No. 6, October 1999, pp. 999-1001. (C) Indian Academy of Sciences.

\title{
Influence of phase transformation in Pd hydride on the recovery characteristics of optical hydrogen sensors
}

\author{
Y OKUHARA, Y IMAI, Y NOGUCHI and M TAKATA* \\ Department of Electrical Engineering, Nagaoka University of Technology, 1603-1 Kamitomioka, Nagaoka, \\ Niigata 940-2188, Japan
}

MS received 26 January 1999; revised 2 June 1999

\begin{abstract}
The recovery characteristics of optical hydrogen sensor, using Pd film, have been investigated in order to clarify their distinctive behaviour showing three-stage curve. The obvious two peaks observed in differentiated reflectance as a function of elapsed time during hydrogen desorption were found to correspond to the phase boundaries of Pd hydride. The phase transformation determined by these peaks was consistent with that observed with in situ X-ray diffraction analysis. Moreover, the change in resistivity with hydrogen desorption indicated distinctive recovery curve, which was also connected with the phase transformation. These results clarified that the hydrogen desorption obviously consisted of three-stage process, and the distinctive behaviour was due to the phase transformation of Pd hydride.
\end{abstract}

Keywords. Hydrogen sensor; optical sensing; recovery characteristics; hydrogen desorption; catalysis; palladium.

\section{Introduction}

In the previous paper, the recovery behaviour of the optical hydrogen sensor, using $\mathrm{Pd}$ film, was reported (Okuhara and Takata 1999). It was observed that the change in reflectance with hydrogen desorption indicated distinctive recovery characteristics showing three-stage curve. Moreover, the phase boundary of Pd hydride was determined by the total reflectance change as a function of hydrogen partial pressure, and the correlation between the three-stage hydrogen desorption and the phase transformation of $\mathrm{Pd}$ hydride from $\beta$ - to $\alpha$ phase was mentioned briefly.

In the present work, the relation between the distinctive recovery behaviour and the phase transformation of $\mathrm{Pd}$ hydride through the hydrogen desorption process have been investigated in detail using in situ $\mathrm{X}$-ray diffraction (XRD) analysis, and electrical resistivity measurement.

\section{Experimental}

Experimental details of sample preparation and optical reflectance measurement have been described in the previous report by Okuhara and Takata (1999), wherein the buffer layer of $W$ was thinned so that its resistance could be neglected. The thickness of Pd and W were 100 and $5 \mathrm{~nm}$, respectively. Henceforth, the double-layered film will be referred to as the $\mathrm{Pd} / \mathrm{W}$ film.

\footnotetext{
*Author for correspondence
}

The optical reflectance and the electrical resistivity of the $\mathrm{Pd} / \mathrm{W}$ films were measured simultaneously. The electrical resistivity measurement was carried out by using the four-probe method, with d.c. current of $1 \mathrm{~mA}$. The phase and the lattice spacing of Pd and Pd hydride were estimated by using an X-ray diffractometer (RIGAKU, RINT-1500) cell into which $\mathrm{H}_{2}$ and $\mathrm{Ar}$ could be introduced. All measurements were conducted at room temperature and atmospheric pressure.

\section{Results and discussion}

Figure 1 shows XRD profiles for $\mathrm{Pd} / \mathrm{W}$ film before and after exposing to hydrogen gas, and during hydrogen desorption. A peak assigned to Pd (111) was observed before exposing to hydrogen gas (figure 1a). A peak of $\beta$-Pd hydride (111) $\left(\mathrm{PdH}_{x}, x>0.46\right.$ at $300 \mathrm{~K}$ (Lee and Glosser 1985)) appeared instead of $\mathrm{Pd}$ (111) after hydrogen absorption (figure $1 b$ ). Following the replacement of hydrogen gas by $\mathrm{Ar}$, the peak intensity of $\beta-\mathrm{PdH}_{x}$ decreased, and a peak of $\alpha-\mathrm{PdH}_{x}(x<0.07$ at $300 \mathrm{~K}$ (Lee and Glosser 1985)) appeared with time as shown in figures $1 \mathrm{c}$ and $\mathrm{d}$. Finally, the peak of $\beta-\mathrm{PdH}_{x}$ disappeared and the peak of $\mathrm{Pd}$ remained as shown in figure 1e. These results indicate that $\beta$-Pd hydride film was formed subsequent to the exposure of $\mathrm{Pd}$ film to hydrogen gas, followed by co-existence of $\alpha$ and $\beta$ phases of $\mathrm{Pd}$ hydrides, indicated by the plateau region, and subsequently the formation of $\alpha-\mathrm{Pd}$ hydride and $\mathrm{Pd}$ metal resulted with continued desorption process. The observed 
shift in diffraction angle for $\beta$-Pd hydride during hydrogen desorption process has been attributed to the decrease in lattice spacing of $\beta$-Pd hydride with a diminution in hydrogen content. Moreover, the decrease in lattice spacing of Pd (111), compared with virgin Pd film, was observed after hydrogen desorption (figure 1e). It is known that the spacing of lattice planes parallel to the surface of the specimen decreased with the applied tensile stresses parallel to the surface due to the Poisson contractions (Cullity 1978). Hence, the observed decrease in lattice spacing of Pd (111) might be due to the residual tensile stress parallel to the Pd surface by the lattice expansion-contraction process. Figure 2 shows change in lattice spacing of Pd (111) with the increase in the number of hydrogen absorption-desorption cycles. After the initial drastic decrease in the lattice spacing for the first cycle, the lattice spacing gradually increased with the

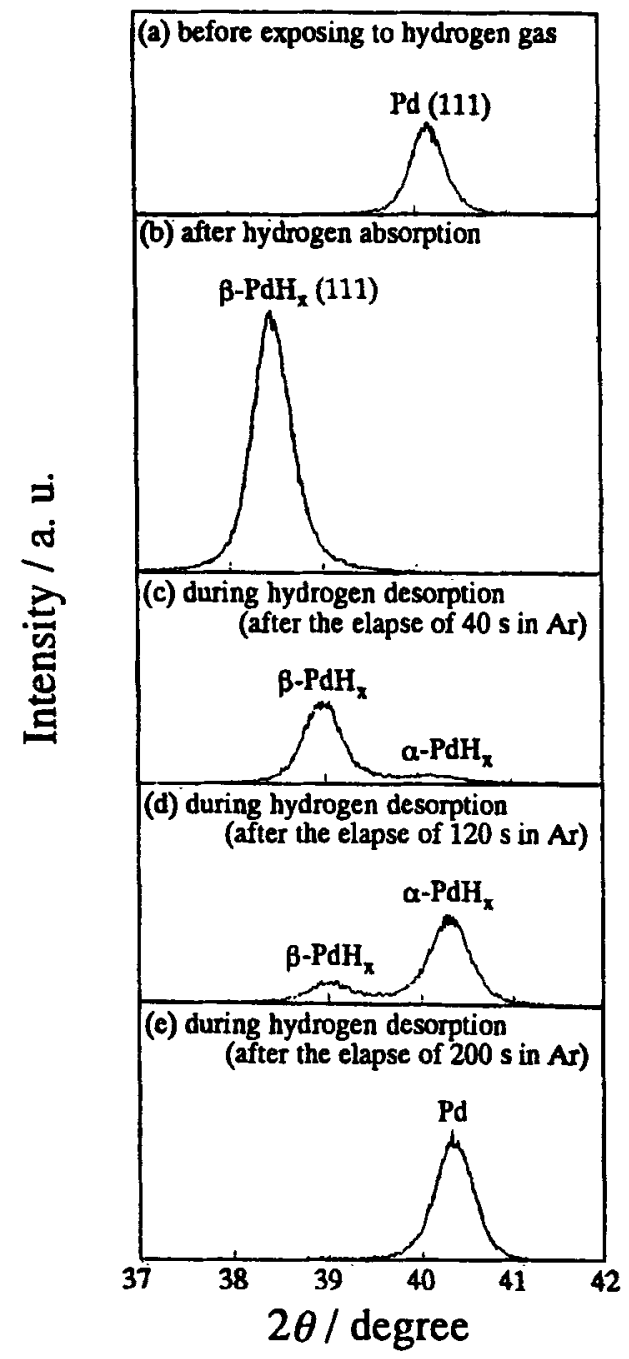

Figure 1. X-ray $(\mathrm{Cu} \mathrm{K} \alpha)$ diffraction profiles of $\mathrm{Pd} / \mathrm{W}$ film. a. Before exposing to hydrogen gas. b. After hydrogen absorption. c.-e. During hydrogen desorption. XRD profiles were measured about $40 \mathrm{~s}, 120 \mathrm{~s}$ and $200 \mathrm{~s}$ after replacement of hydrogen gas by $\mathrm{Ar}$. increase in the number of cycles. This increase in lattice spacing is considered to correspond to relaxation of the residual stress. In the previous paper, it was reported that distinction of recovery characteristics with three-stage curve became clearer with increasing the number of adsorption-desorption cycles (Okuhara and Takata 1999). Since the relaxation of stress causes the sharpening of pressure concentration isotherm (Nicolas et al 1986), the progress of relaxation might induce the more distinctive recovery curve.

Figure 3a presents the change in reflectance for Pd film with hydrogen absorption and desorption as a function of time. While the hydrogen absorption into Pd film resulted in rapid decrease in reflectance to the saturation point within $3 \mathrm{~s}$, the hydrogen desorption from $\mathrm{Pd}$ hydride gradually increased the reflectance to the initial level. The distinctive recovery curve showing three stages was observed by Okuhara et al (to be published). Figure $3 \mathrm{a}^{\prime}$ shows the reflectance differentiated with respect to time during hydrogen desorption process. This curve had obvious two peaks, which well reflected the recovery behaviour with three-stage curve.

The relation between the phase transformation shown in figures $1 \mathrm{~b}-\mathrm{e}$ and three-stage curve indicated in figure $3 \mathrm{a}$ should be clarified by determination of the phase boundary in hydrogen desorption process. Figure $3 b$ shows the dependence of total reflectance change $(-\Delta R)$ on hydrogen pressure. The method for measuring this relation, and the reason for hysteresis between absorption and desorption of hydrogen was described in the previous paper (Okuhara and Takata 1999). Since this result was similar to pressure-concentration isotherm (Lee et al 1985), the phase of Pd hydride can be assigned, as shown in figure $3 b$. Thus, it can be seen that the peak positions in figure $3 a^{\prime}$ correspond to the phase boundaries distinguished in figure $3 \mathrm{~b}$. Hence the phase transformations, as

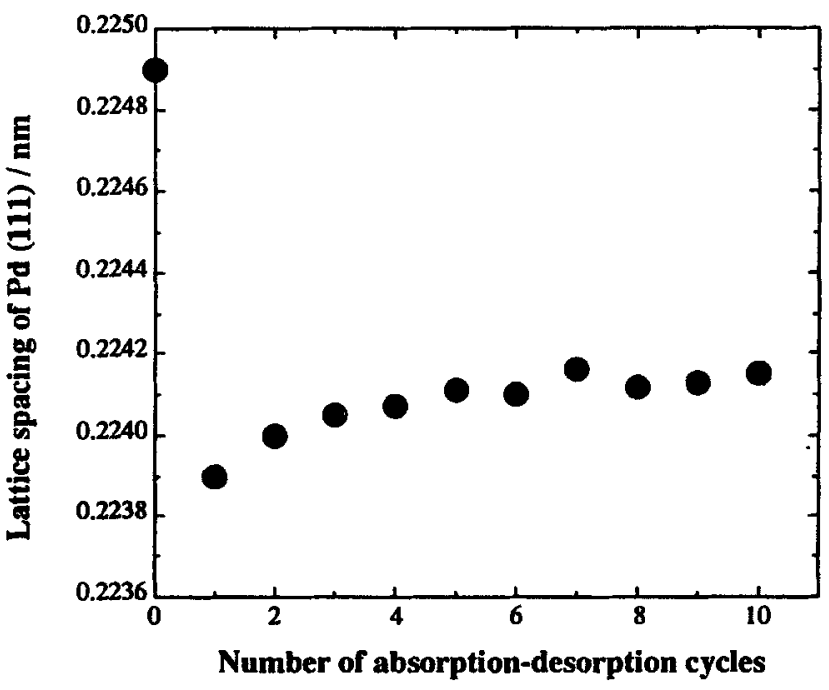

Figure 2. Change in lattice spacing of $P d$ (111) with increasing number of hydrogen absorption-desorption cycles. 


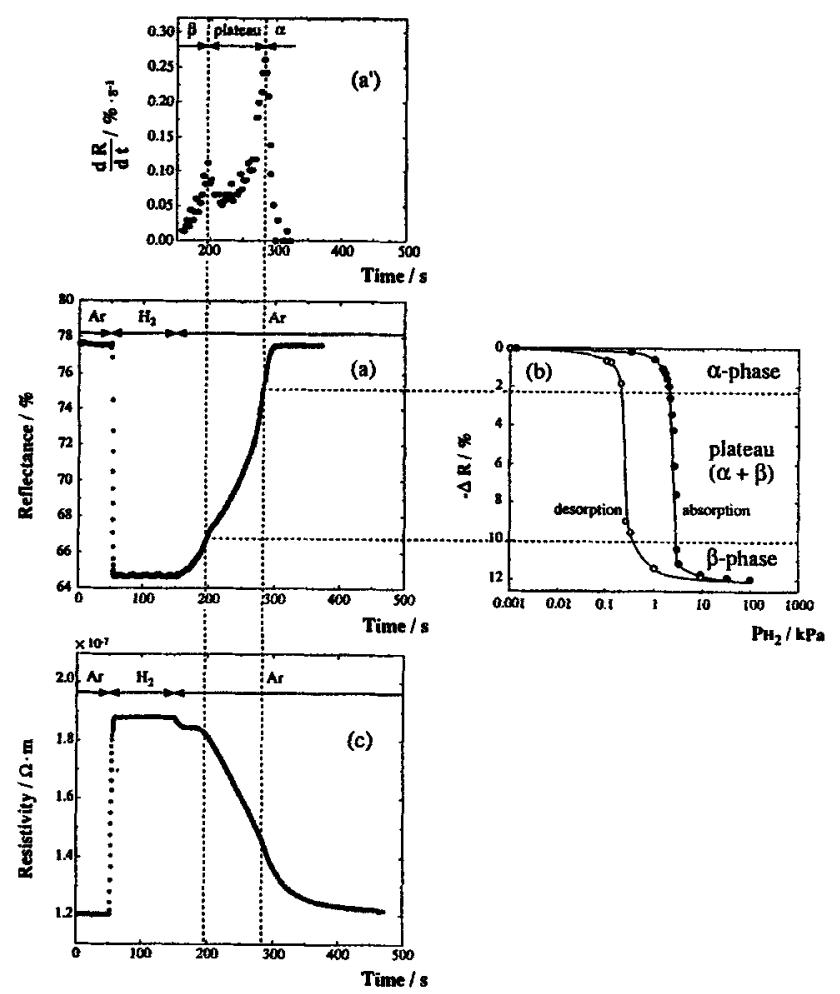

Figure 3. Relation between recovery characteristics and phase transformation of $\mathrm{Pd}$ hydride. a. Change in reflectance for $\mathrm{Pd} / \mathrm{W}$ with hydrogen absorption and desorption at room temperature. $\mathbf{a}^{\prime}$. Differentiated reflectance for Pd hydride thin film with hydrogen desorption. b. Total reflectance change as a function of hydrogen partial pressure. c. Change in resistivity for $\mathrm{Pd} / \mathrm{W}$ with hydrogen absorption and desorption.

a function of elapsed time during hydrogen desorption, can be determined from the peaks shown in figure $3 a^{\prime}$, and were shown to be consistent with the result of XRD analysis shown in figure 1 . Thus, these results proved the previous interpretation of Okuhara and Takata (1999) wherein the three-stage hydrogen desorption had been correlated to the phase transformation of Pd hydride.

Figure $3 \mathrm{c}$ presents the change in resistivity for $\mathrm{Pd} / \mathrm{W}$ film with hydrogen absorption and desorption as a function of time. The resistivity rapidly increased with hydrogen absorption, and gradually decreased with hydrogen desorption. The ratio of resistivity of $\mathrm{PdH}_{x}$ after hydrogen absorption to the initial one of the "hydrogen free'-Pd was about $1 \cdot 6$, which corresponded to the relative resistance change at $\mathrm{H} / \mathrm{Pd}$ atomic ratio of about 0.6 (Barton et al 1963). This result also indicates that Pd film transformed to $\beta-\mathrm{PdH}_{x}$ film after being exposed to hydrogen gas. The recovery behaviour of resistivity slightly differed from that of reflectance and showed a bulge at the initial stage. It is known that the presence of a bulge in the relation between relative resistance change and $\mathrm{H} / \mathrm{Pd}$ atomic ratio appears around the phase boundary between $\beta-\mathrm{PdH}_{x}$ and plateau region (Lewis 1967). Since figures $3 \mathrm{a}$ and $\mathrm{c}$ were obtained simultaneously, the relation between the phase of $\mathrm{PdH}_{x}$ and the elapsed time, distinguished in figure $3 a^{\prime}$, can be applied to figure $3 c$, and therefore the bulge decidedly appeared around the phase boundary between $\beta-\mathrm{PdH}_{x}$ and plateau region. Moreover, the slope of the recovery curve of resistivity changed at $t=280 \mathrm{~s}$, which corresponded to the phase transformation from plateau region to $\alpha-\mathrm{PdH}_{x}$. These results further support the contention that the distinctive hydrogen desorption was due to the phase transformation of Pd hydride.

\section{Conclusion}

The relation between the recovery characteristics of optical hydrogen sensor showing three-stage curve, and the phase transformation of Pd hydride was investigated. In situ XRD analysis revealed that $\beta$-Pd hydride was formed from Pd film with hydrogen adsorption, and was transformed to metal phase through plateau region during hydrogen desorption. The obvious two peaks were observed in differentiated reflectance as a function of elapsed time during hydrogen desorption. These peaks corresponded to the phase boundaries estimated from the total reflectance change as a function of hydrogen partial pressure. The phase transformation determined from these peaks was consistent with that observed with in situ XRD analysis. Moreover, the recovery curve of resistivity of Pd hydride film indicated distinctive behaviour, which was also relevant to the phase transformation. These results strongly support the contention that the hydrogen desorption consisted of three-stage process and further clarified that the three-stage hydrogen desorption was due to the phase transformation of Pd hydride.

\section{Acknowledgement}

This work was supported by a Grant-in-Aid for Science Research from the Ministry of Education, Science, Sports and Culture (No. 07555192 and 10875127).

\section{References}

Barton J C, Lewis F A and Woodward I 1963 Trans. Faraday Soc. 591201

Cullity B D 1978 Elements of X-ray diffraction (Massachusetts: Addison-Wesley Pub. Co.) Second edn, chapter 16

Lee $\mathrm{M}$ and Glosser R 1985 J. Appl. Phys. 575236

Lewis F A 1967 The palladium hydrogen system (New York: Academic Press) chap. 4

Nicolas M, Dumoulin L and Burger J P 1986 J. Appl. Phys. 603125 Okuhara Y and Takata M 1999 Bull. Mater. Sci. 2285 